Check for updates

Cite this: J. Mater. Chem. C, 2020, 8, 9502

Received 28th April 2020

Accepted 1st July 2020

DOI: $10.1039 / \mathrm{dOtc02075c}$

rsc.li/materials-c

\section{Prolonged and efficient near-infrared photoluminescence of a sensitized organic ytterbium-containing molecular composite $\dagger$}

\author{
Chen Lyu, (D) a Hongfei Li, ${ }^{a}$ Peter B. Wyatt, ${ }^{b}$ William P. Gillin (D) *ac and \\ Huanqing $\mathrm{Ye}^{\star \mathrm{ac}}$
}

\begin{abstract}
Organic prolonged luminescent materials have attracted attention with various candidates reported providing both UV and visible emission for applications in bio-imaging, light storage, security, etc. However, there is a lack of prolonged near-infrared (NIR) emitting materials, which are highly desirable for many of the proposed applications. NIR-emitting organic lanthanide(III) molecules have suitable wavelengths, but all of them are unsuitable for persistent luminescence due to weak sensitization and the restricted intrinsic ion radiative lifetimes. Herein, this paper demonstrates efficiently sensitized organic $\mathrm{Yb}(\mathrm{III})$ compounds with prolonged emission lifetimes up to $\sim 0.3 \mathrm{~s}$ at $1 \mu \mathrm{m}$, far exceeding the intrinsic $\mathrm{Yb}^{3+}$ emission lifetime of $\sim 1 \mathrm{~ms}$. The dynamic equilibrium is studied to demonstrate that this prolonged emission is caused by energy transfer from long-lived organic triplet excitons. Experiment and simulation results suggest a new route to develop bright and ultralong-lived $1 \mu \mathrm{m}$ emitting materials that could be coupled with other existing organic persistent luminescence materials to shift their emission in to the infrared.
\end{abstract}

There has been considerable interest in the engineering of interor intra-molecular interactions to trap triplet excitons and to use this to demonstrate organic prolonged phosphorescence. ${ }^{1-7}$ Since triplet excitons in organics mainly result from inter-system crossing (ISC) with a small energy separation from the triplets, the reported phosphoresce usually only red-shifts in the visible, e.g. to $\sim 500 \mathrm{~nm}$ to $600 \mathrm{~nm}$ compared to UV-visible fluorescence centred at a wavelength of $\sim 300 \mathrm{~nm}$ to $400 \mathrm{~nm} .{ }^{4-6}$ However, this UV-visible luminescence is not ideal for many of the proposed applications and NIR light, at a wavelength of $\sim 1 \mu \mathrm{m}$ (energy of

\footnotetext{
${ }^{a}$ Materials Research Institute and School of Physics and Astronomy, Queen Mary University of London, Mile End Road, London E1 4NS, UK.

E-mail: h.ye@qmul.ac.uk,w.gillin@qmul.ac.uk

${ }^{b}$ Materials Research Institute and School of Biological and Chemical Sciences, Queen Mary University of London, Mile End Road, London E1 4NS, UK ${ }^{c}$ Chromosol Ltd, The Walbrook Building, 25 Walbrook, London, EC4N 8A, UK $\dagger$ Electronic supplementary information (ESI) available. See DOI: 10.1039/ d0tc02075c
}

$\sim 1.1 \mathrm{eV}$ ), would be preferred in many biological applications, due to the high transmission in biological systems at this wavelength and in anti-counterfeiting applications as NIR is not visible to the naked eye. ${ }^{8-15}$

Organic $\mathrm{Yb}$ (III) materials with an organic chromophore that strongly absorbs light to sensitize $\mathrm{Yb}^{3+}$ ions can give bright and sharp $1 \mu \mathrm{m}$ emission under low-power photoexcitation. Various organic $\mathrm{Yb}$ (III) complexes or hybrid organic-conjugated $\mathrm{Yb}^{3+}$ doped nanoparticles have been demonstrated to provide sensitized $\mathrm{Yb}^{3+}$ luminescence. ${ }^{16,17}$ However, a general problem is that $\mathrm{Yb}^{3+}$ emission can be severely quenched by high-energy organic vibrations, e.g. $\mathrm{C}-\mathrm{H}, \mathrm{N}-\mathrm{H}$ or $\mathrm{O}-\mathrm{H}$ bonds, and this causes luminescence lifetimes as short as nanoseconds to microseconds with poor efficiencies of $<0.1 \% .^{18,19}$ Even though there are effective protective methods to eliminate quenching, the sensitized $\mathrm{Yb}^{3+}$ emission lifetime is capped by the intrinsic radiative lifetime which is usually shorter than $2 \mathrm{~ms}$ and far from the demands of persistent PL.

Our approach utilizes an organic chromophore to sensitize an organic $\mathrm{Yb}$ (III) molecule in a molecular composite thin film. The organic $\mathrm{Yb}$ (III) molecule we use is a chelate, $\mathrm{Yb}$ (F-TPIP) that has a $\mathrm{Yb}^{3+}$ ion incorporated with tetrakis-(pentafluorophenyl)imidodiphosphinate, F-TPIP ${ }^{-}$ligands, and it is inert to visible light. ${ }^{20}$ The organic chromophore we employ is the zinc salt of 2-(tetrafluoro-2-hydroxyphenyl)tetrafluorobenzothiazole, $\mathrm{Zn}(\mathrm{F}-\mathrm{BTZ})_{2}$, which has strong absorption of blue light. ${ }^{21}$ The chemical properties of the two individual materials have been well studied in previous work. ${ }^{20,21}$

The $405 \mathrm{~nm}$ light photoexcites Zn(F-BTZ) ${ }_{2}$ to give the Zn(F-BTZ) emission broadly spanning from $\sim 450 \mathrm{~nm}$ to $900 \mathrm{~nm}$ and sensitizes the sharp $\mathrm{Yb}^{3+}$ emission centred at $975 \mathrm{~nm}$ due to the $\mathrm{Yb}^{3+}:{ }^{2} \mathrm{~F}_{5 / 2} \rightarrow{ }^{2} \mathrm{~F}_{7 / 2}$ transition, shown in Fig. 1. The excitation spectrum for the $\mathrm{Yb}^{3+}$ emission recorded at $1005 \mathrm{~nm}$ matches features of the $\mathrm{Zn}(\mathrm{F}-\mathrm{BTZ})_{2}$ excitation spectrum at $500 \mathrm{~nm}$, indicating a sensitization effect of $\mathrm{Zn}(\mathrm{F}-\mathrm{BTZ})_{2}$ onto $\mathrm{Yb}^{3+}$ excitations (i.e. light is being absorbed by the $\mathrm{Zn}(\mathrm{F}-\mathrm{BTZ})_{2}$ and the energy is then transferred to the $\mathrm{Yb}^{3+}$ ions from which it is 


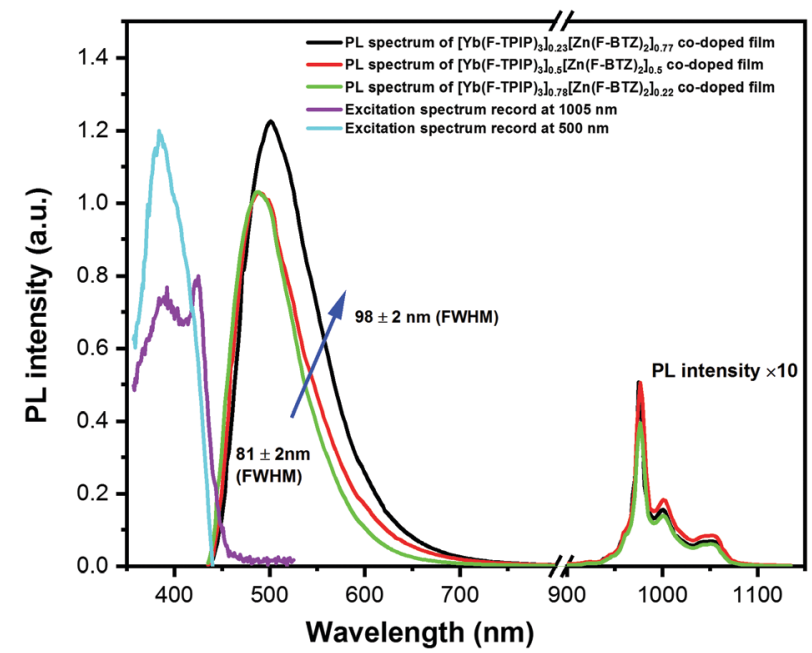

Fig. $1 \mathrm{PL}$ spectra of co-doped films of $\left[\mathrm{Yb}(\mathrm{F}-\mathrm{TPIP})_{3}\right]_{x}\left[\mathrm{Zn}(\mathrm{F}-\mathrm{BTZ})_{2}\right]_{1-x}$ $(x=0.23,0.5,0.78)$ and the excitation spectrum of a co-doped film of $\left[\mathrm{Yb}(\mathrm{F}-\mathrm{TPIP})_{3}\right]_{0.5}\left[\mathrm{Zn}(\mathrm{F}-\mathrm{BTZ})_{2}\right]_{0.5}$. The PL spectra (black, red and green lines) were measured at photoexcitation using a $405 \mathrm{~nm}$ laser. The excitation spectra (violet line and light blue line) were recorded at an emission wavelength of $1005 \mathrm{~nm}$ and $500 \mathrm{~nm}$, respectively. Both the excitation and $\mathrm{PL}$ spectra are optically corrected by the responsivity of photon detectors and spectrometer gratings.

emitted). Previous studies ${ }^{22}$ have shown abundant triplet excitons in $\mathrm{Zn}(\mathrm{F}-\mathrm{BTZ})_{2}$ and its phosphorescence is centred around $550 \mathrm{~nm}$ extending to the NIR beyond $900 \mathrm{~nm}$. This implies the phosphorescence band slightly overlaps the $\mathrm{Yb}^{3+}$ absorption which makes the sensitization from the triplet states possible, probably explaining the observation that higher $\mathrm{Yb}(\mathrm{F}-\mathrm{TPIP})_{3}$ doping concentrations selectively quench the phosphorescence to narrow the FWHM of the $\mathrm{Zn}(\mathrm{F}-\mathrm{BTZ})_{2}$ emission (Fig. 1).

Fig. 2(a) shows the time-resolved PL (TRPL) of excited $\mathrm{Yb}^{3+}$ ions recorded at $975 \mathrm{~nm}$. The directly excited $\mathrm{Yb}^{3+}$ emission lifetime is $0.46 \pm 0.12 \mathrm{~ms}$, indicating an internal quantum efficiency of $46 \%$, using a reported intrinsic $\mathrm{Yb}^{3+}$ radiative lifetime of $\sim 1 \mathrm{~ms}$ in $\mathrm{Yb}(\mathrm{F}-\mathrm{TPIP})_{3}{ }^{23}$ The sensitized $\mathrm{Yb}^{3+}$ emission lifetime becomes distinctly longer than those obtained by direct excitation into the $\mathrm{Yb}^{3+}:^{2} \mathrm{~F}_{5 / 2}$ level. Four lifetimes are obtained by fitting with their component percentages. The shortest lifetime $\left(\tau_{1}\right)$ of $0.22 \pm 0.11 \mathrm{~ms}$ with a component percentage of $6.8 \%$ is believed to result from $\mathrm{Yb}^{3+}$ ions that are close to residual quenching centres in the film. The longer lifetime $\left(\tau_{2}\right)$ of $0.68 \pm 0.15 \mathrm{~ms}(24.83 \%)$ corresponds to $\mathrm{Yb}^{3+}$ ions distant from quenching centres. The information about quenching centres and protective techniques is discussed in the ESI. $\uparrow$ These demonstrate that the film contains at least two quenching environments, which lead to corresponding internal quantum efficiencies (IQEs) of $21 \%$ and $68 \%$, respectively. Interestingly, the lifetime $\left(\tau_{3}\right)$ of $2.22 \pm 0.5 \mathrm{~ms}(26.59 \%)$ and the longest lifetime $\left(\tau_{4}\right)$ of $10.2 \pm 1.5 \mathrm{~ms}(45.7 \%)$ are considerably prolonged compared to that for direct excitation and exceed the intrinsic $\mathrm{Yb}^{3+}$ radiative lifetime $(1 \mathrm{~ms})$. Also, these two prolonged lifetimes are comparable to the long phosphorescence lifetimes of $2.2 \pm 0.4 \mathrm{~ms}$ and $11.9 \pm 2.7 \mathrm{~ms}$ for the triplet emission at $600 \mathrm{~nm}$ (used to reduce the singlet

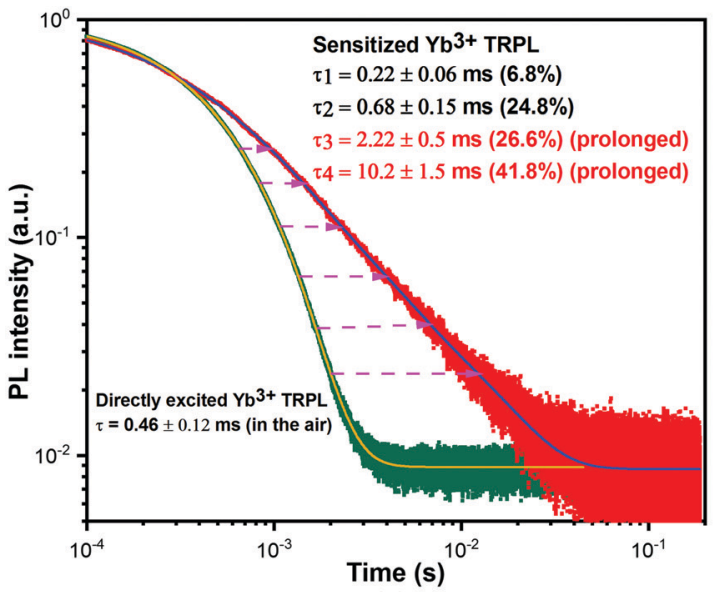

(a)

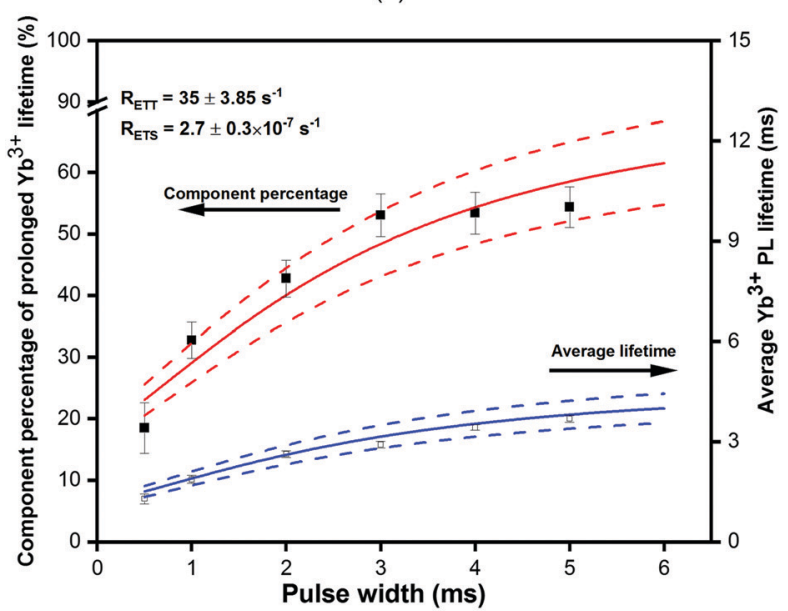

(b)

Fig. 2 (a) Directly excited and sensitized time-resolved room-temperature $\mathrm{PL}$ spectra of the co-doped film of $\left[\mathrm{Yb}(\mathrm{F}-\mathrm{TPIP})_{3}\right]_{0.5}\left[\mathrm{Zn}(\mathrm{F}-\mathrm{BTZ})_{2}\right]_{0.5}$. The green dots show the directly excited TRPL of the composite film and the red dots show the sensitized TRPL. The orange and blue curves are obtained by fitting the decay traces using exponential decay functions. (b) The component percentages of the prolonged $\mathrm{Yb}^{3+} \mathrm{PL}$ lifetimes (solid squares) and the average lifetime of $\mathrm{Yb}^{3+} \mathrm{PL}$ (open squares) evolved with photoexcitation pulse widths. The simulated traces for the component percentages and the average $\mathrm{Yb}^{3+} \mathrm{PL}$ lifetimes are presented by a red and blue curve, respectively. The error bars of the simulation are given by the dashed lines.

contribution to the decay curve) (Fig. S7 in the ESI $\dagger$ ), which implies that energy transfer from the triplets is probably responsible.

To further investigate those prolonged $\mathrm{Yb}^{3+}$ emission lifetimes, we varied the pulse-width of a $405 \mathrm{~nm}$ laser photoexcitation to manipulate the percentages of the four lifetime components, shown in Fig. 2(b). With a longer pulse width, the population of triplet states around each $\mathrm{Yb}(\mathrm{F}-\mathrm{TPIP})_{3}$ molecule will accumulate to increase the probability to sensitize the $\mathrm{Yb}^{3+}$ ion. Consequently, the total percentage of the prolonged lifetimes of $\tau_{3}$ and $\tau_{4}$, which appear to be relevant to triplet states, increases with longer pulse widths. The increase in the total percentage makes the average lifetime for the obtained $\mathrm{Yb}^{3+}$ emission become longer with extending pulse width. We resolved a set of rate equations to understand the relationship between the 


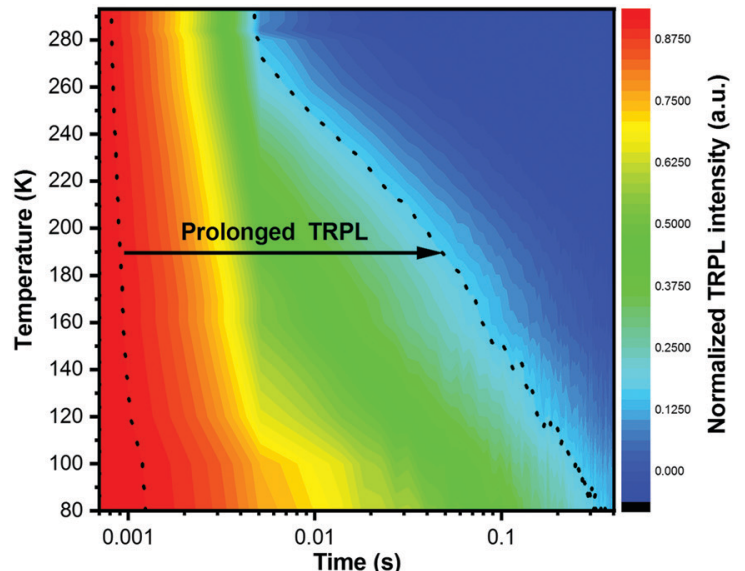

(a)

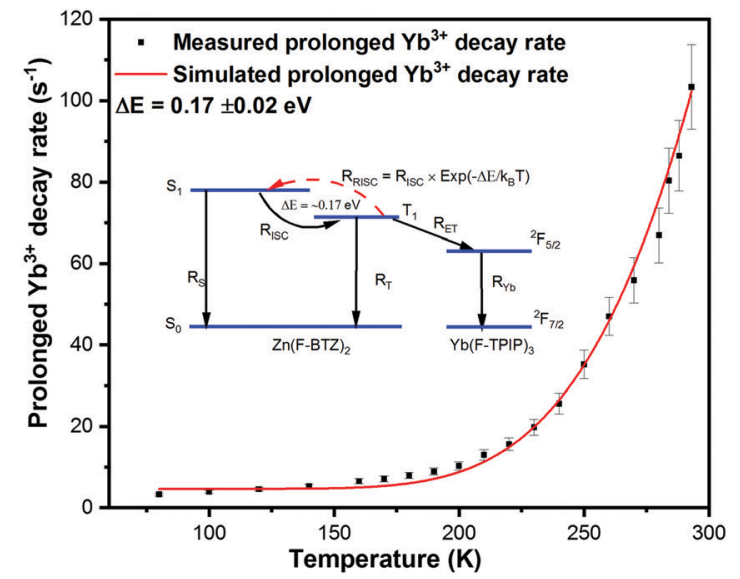

(b)

Fig. 3 (a) Temperature dependent sensitized $\mathrm{Yb}^{3+} \mathrm{TRPL}$ of a co-doped film of $\left[\mathrm{Yb}(\mathrm{F}-\mathrm{TPIP})_{3}\right]_{0.5}\left[\mathrm{Zn}(\mathrm{F}-\mathrm{BTZ})_{2}\right]_{0.5}$ at $975 \mathrm{~nm}$. (b) The measured and simulated temperature-dependent prolonged $\mathrm{Yb}^{3+}$ decay rates, which resulted from the introduction of a reverse ISC process into the analytical solution of the rate equations shown in the ESI. $\dagger$

prolonged $\mathrm{Yb}^{3+}$ excitations and triplet states. The construction and deduction of rate equations contain two different time regimes. More details can be found in the ESI. $\dagger$

The calculation shows that the decay rate for the $\mathrm{Yb}^{3+}:^{2} \mathrm{~F}_{5 / 2}$ level is a combination of both the triplet decay rate and the intrinsic decay rate of excited $\mathrm{Yb}^{3+}$ ions. The $\mathrm{e}^{-\left(R_{\mathrm{ETT}}+R_{\mathrm{T}}\right) \times t_{2}}$ term (see eqn (S17) in the ESI $\dagger$ ) is a decay process for the $\mathrm{Yb}^{3+}$ emission that follows the decay rate of the $\mathrm{Zn}(\mathrm{F}-\mathrm{BTZ})_{2}$ triplet states. This explains why those prolonged $\mathrm{Yb}^{3+}$ emission lifetimes are similar to the persistent phosphorescence lifetimes of the triplet states. As mentioned above, the presence of either $\mathrm{Yb}$ (F-TPIP $)_{3}$ or Y(F-TPIP) $)_{3}$ makes no difference to the phosphorescence lifetimes within the range of the experimental error bars. Hence, it is reasonable to use the maximum value of the error bars to estimate the ET rate, which is $R_{\mathrm{ETT}}=\sim 35 \mathrm{~s}^{-1}$ (see Fig. S7 in the ESI $\dagger$ ). Moreover, the calculation can give the average $\mathrm{Yb}^{3+}$ emission lifetime as a function of the photoexcitation pulse width which is shown as the solid lines in Fig. 2(b), with the calculated time-resolved component percentage of the prolonged $\mathrm{Yb}^{3+}$ emission lifetime. The simulation is seen to match the experimental data.

We have used the spectral overlap integral between the triplet phosphorescence spectrum and the $\mathrm{Yb}^{3+}$ absorption spectrum to estimate a Förster resonant energy transfer (FRET) rate, $R_{\mathrm{FRET}}$, which is a conventional method to quantify the Förster energy transfer. The detail of the FRET rate calculation can be found in the ESI. $\dagger$ There are always considerable uncertainties in the parameters used in FRET calculations, not least including the donor quantum yield, orientation factor and the donor-acceptor separation. However, by taking reasonable estimations of the quantum yield as $10 \%$, and the donoracceptor distance from $7 \AA$ (the radius of single Yb(F-TPIP) ${ }_{3}$ molecule) to $10 \AA$ (the maximum working distance of FRET), ${ }^{20}$ the calculated $R_{\mathrm{FRET}}$ varies from $64 \mathrm{~s}^{-1}$ to $5 \mathrm{~s}^{-1}$, which are in agreement with our $R_{\mathrm{ETT}}$. It is noted that eqn (S21) and (S22) in the ESI $\dagger$ indicate the quantum yield of the energy donor $Q_{\mathrm{D}}$ would only linearly scale the value of $R_{\mathrm{FRET}}$. Hence, as limited by the maximum and minimum value of $Q_{\mathrm{D}}$, the value of $R_{\mathrm{FRET}}$ is consistent with $R_{\mathrm{ETT}}$ to within one order of magnitude.

We present two possible processes to explain the prolonged $\mathrm{Yb}^{3+}$ excitations. Firstly, since the low ET rate keeps triplet excitons long-lived, if an excited $\mathrm{Yb}^{3+}$ ion relaxes to the ground state, it is possible for a neighbouring triplet exciton to re-excite that de-excited $\mathrm{Yb}^{3+}$ ion. Also, long-lived triplet excitons can diffuse via intermolecular interactions among Zn(F-BTZ) $)_{2}$ molecules to reach such an $\mathrm{Yb}^{3+}$ ion. These two routes maintain the population level of excited $\mathrm{Yb}^{3+}$ ions to overcome the intrinsic $\mathrm{Yb}^{3+}$ decay rate.

We have also measured the temperature dependent sensitized$\mathrm{Yb}^{3+}$ TRPL at $975 \mathrm{~nm}$ in a co-doped film of $\left[\mathrm{Yb}(\mathrm{F}-\mathrm{TPIP})_{3}\right]_{0.5}$ $[\mathrm{Zn}(\mathrm{F}-\mathrm{BTZ})]_{0.5}$. The contour-colour display of the spectra is shown in Fig. 3(a). It can be clearly seen that prolonged $\mathrm{Yb}^{3+}$ emission becomes dominant with components of $334.5 \pm 30.1 \mathrm{~ms}(87.9 \%)$ with $74.4 \pm 5.9 \mathrm{~ms}(11.3 \%)$ at $80 \mathrm{~K}$. The values of the fitted lifetimes and their component percentages are shown in Table S3 (ESI + ). This prolonged $\mathrm{Yb}^{3+}$ PL implies that a triplet quenching process is suppressed at low temperature. We believe this process is thermally activated reverse-intersystem-crossing (RISC). Using the simple rate model shown in the inset to Fig. $3(\mathrm{~b})$ we can fit the data and obtain an energy gap of $0.17 \pm 0.02 \mathrm{eV}$ between the triplet state $\left(\mathrm{T}_{1}\right)$ and the singlet state $\left(\mathrm{S}_{1}\right)$ of $\mathrm{Zn}(\mathrm{F}-\mathrm{BTZ})_{2}$. The fitted energy gap is of the order of the singlet-triplet energy separation in the chromophore. The result implies the use of a chromophore material with a larger singlet-triplet energy separation would provide a route to exceptionally prolonged room temperature $\mathrm{Yb}^{3+}$ PL lifetimes using this approach.

\section{Conclusions}

In summary, composite thin films are fabricated to produced sensitized $\mathrm{Yb}^{3+} 1 \mu \mathrm{m}$ photoluminescence. Sensitized timeresolved photoluminescence measurement indicates that the sensitized $\mathrm{Yb}^{3+}$ lifetime is prolonged to exceed its intrinsic 
lifetime. Simulation of excited state dynamic suggests the prolonged lifetime is due to the energy transfer from long-lived triplet. Additionally, our work demonstrates a potential route to develop bight and ultra-long lived $1 \mu \mathrm{m}$ emitting material that could be coupled to some known persist luminescence materials to shift their emission wavelength to infrared.

\section{Methods}

Details of methods, materials and optical measurement techniques can be found in the ESI. $\dagger$ HFTPIP and HFBTZ ligands were supplied by Chromosol Ltd. $\mathrm{YbCl}_{3} \cdot\left(\mathrm{H}_{2} \mathrm{O}\right)_{6}$ (metals basis 99.99\% purity) was purchased from Alfa Aesar. $\mathrm{Yb}^{3+}$ salt and the HFTPIP ligand were reacted in methanol:ethanol solution to produce $\mathrm{Yb}(\mathrm{F}-\mathrm{TPIP})_{3}$ precipitates. $\mathrm{KOH}$ and the HFBTZ ligand were used to form KFBTZ and then reacted with a $\mathrm{Zn}^{2+}$ salt to precipitate $\mathrm{Zn}(\mathrm{F}-\mathrm{BTZ})_{2}$. The reaction condition to make $\mathrm{Yb}(\mathrm{F}-\mathrm{TPIP})_{3}$ and $\mathrm{Zn}(\mathrm{F}-\mathrm{BTZ})_{2}$ are routine and have been reported. ${ }^{20,21} \mathrm{~A}$ train vacuum purification system was used to purify the materials before the use. The display of Fig. 2(a) uses logarithmic scales for both the $x$-axis and $y$-axes, to fully visualize the details of the decay curves over a wide range time regime, which cannot be seen with a linear $x$-axis. The composite of two materials in thin films are fabricated using a co-evaporation technique using a Kurt J. Lesker SPECTROS deposition system in a 10 K-class cleanroom. A Hamamatsu R5509-72 nitrogen-cooled detector with a Horiba Triax 550 spectrometer was used to collect and detect photoluminescence signals. Multiple exponential functions are used to fit time-resolved decay curves to obtain TRPL lifetimes. All the film samples were covered by a $100 \mathrm{~nm}$ layer of thermally grown aluminium film. The low temperature measurements were taken using an Oxford Instrument continuous flow cryostat.

\section{Conflicts of interest}

There are no conflicts to declare.

\section{Acknowledgements}

CL and HL are financially supported by the China Scholarship Council and Queen Mary University of London. WPG acknowledges financial support from EPSRC (EP/L020114/1 and EP/P007767/1).

\section{Notes and references}

1 Y. Su, S. Z. F. Phua, Y. Li, X. Zhou, D. Jana, G. Liu, W. Q. Lim, W. K. Ong, C. Yang and Y. Zhao, Sci. Adv., 2018, 4, eaas9732.
2 S. Xu, R. Chen, C. Zheng and W. Huang, Adv. Mater., 2016, 28, 9920-9940.

3 Q. Miao, C. Xie, X. Zhen, Y. Lyu, H. Duan, X. Liu, J. V. Jokerst and K. Pu, Nat. Biotechnol., 2017, 35, 1102.

4 L. Gu, H. Shi, L. Bian, M. Gu, K. Ling, X. Wang, H. Ma, S. Cai, W. Ning and L. Fu, Nat. Photonics, 2019, 13, 406.

5 Z. An, C. Zheng, Y. Tao, R. Chen, H. Shi, T. Chen, Z. Wang, H. Li, R. Deng and X. Liu, Nat. Mater., 2015, 14, 685.

6 Z. Yang, Z. Mao, X. Zhang, D. Ou, Y. Mu, Y. Zhang, C. Zhao, S. Liu, Z. Chi and J. Xu, Angew. Chem., Int. Ed., 2016, 55, 2181-2185.

7 W. Zhao, T. S. Cheung, N. Jiang, W. Huang, J. W. Y. Lam, X. Zhang, Z. He and B. Z. Tang, Nat. Commun., 2019, 10, 1595.

8 J. Feng and H. Zhang, Chem. Soc. Rev., 2013, 42, 387-410. 9 A. D. Aløo, A. Bourdolle, S. Brustlein, T. Fauquier, A. Grichine, A. Duperray, P. L. Baldeck, C. Andraud, S. Brasselet and O. Maury, Angew. Chem., Int. Ed., 2012, 51, 6622-6625.

10 M. H. Futscher, A. Rao and B. Ehrler, ACS Energy Lett., 2018, 3, 2587-2592.

11 W. Shockley and H. J. Queisser, J. Appl. Phys., 1961, 32, 510-519.

12 M. B. Smith and J. Michl, Chem. Rev., 2010, 110, 6891-6936.

13 A. Rao and R. H. Friend, Nat. Rev. Mater., 2017, 2, 17063.

14 N. J. L. K. Davis, J. R. Allardice, J. Xiao, A. J. Petty, N. C. Greenham, J. E. Anthony and A. Rao, J. Phys. Chem. Lett., 2018, 9, 1454-1460.

15 R. B. Anderson, S. J. Smith, P. S. May and M. T. Berry, J. Phys. Chem. Lett., 2014, 5, 36.

16 J.-C. G. Bünzli, Chem. Rev., 2010, 110, 2729-2755.

17 I. Hernández, Y. X. Zheng, M. Motevalli, R. H. C. Tan, W. P. Gillin and P. B. Wyatt, Chem. Commun., 2013, 49, 1933-1935.

18 C. Bischof, J. Wahsner, J. Scholten, S. Trosien and M. Seitz, J. Am. Chem. Soc., 2010, 132, 14334-14335.

19 K. Binnemans, Chem. Rev., 2009, 109, 4283-4374.

20 P. B. Glover, A. P. Bassett, P. Nockemann, B. M. Kariuki, R. Van Deun and Z. Pikramenou, Chem. - Eur. J., 2007, 13, 6308-6320.

21 Z. Li, A. Dellali, J. Malik, M. Motevalli, R. M. Nix, T. Olukoya, Y. Peng, H. Ye, W. P. Gillin, I. Hernández and P. B. Wyatt, Inorg. Chem., 2013, 52, 1379-1387.

22 J. Hu, P. B. Wyatt, W. P. Gillin and H. Ye, J. Phys. Chem. Lett., 2018, 9, 2022-2024.

23 H. Ye, V. Bogdanov, S. Liu, S. Vajandar, T. Osipowicz, I. Hernández and Q. Xiong, J. Phys. Chem. Lett., 2017, 8, 5695-5699. 